\title{
Experimental curriculum of informatics for 11 year old children
}

\author{
Andrej Blaho \\ Ivan Kalas \\ Monika Matusova \\ Comenius University \\ Bratislava \\ Slovak Republic
}

\begin{abstract}
In the Slovak Republic recently a new type of school has been established in lower secondary education. To make the curriculum attractive regular informatics topics are included, although no one knows what the contents should be. This is a very challenging situation for educational research. In our paper we present a new conception of the subject based on an experiment with 11 year old children. We advocate an open learning project approach: our students should understand that computer use may coming in many different and surprising guises. We present six general curriculum topics and several projects, some of them based on paper and pencil work, others implemented in Comenius Logo for Windows. We plan to use Informatics as a kind of a Trojan horse to change the school-to involve teachers of subjects which have no tradition in using the computer as a tool for learning.
\end{abstract}

Main conference themes: methodologies, learner centred learning

Educational areas: primary education

Study topics:

Secondary keywords: curriculum development, classroom practice, creativity, logo 


\section{INTRODUCTION}

In upper secondary school each student has to take a course in informatics for at least one year. Quite often such a course just consists of teaching programming. A similar tendency has been reported from other Central and Eastern European countries, see [1]. The reasons for this are not of interest here, but it is obvious that outdated equipment is one of them. This 8-bit equipment was often distributed with only Basic and Pascal as software.

Among other changes in our society, a new type of school is being established under the name classical secondary school covering both lower and upper secondary education. Many ambitious primary schools cooperate with nearby upper secondary schools to offer coherent education for pupils from 11 to 18 years old. Principals of these schools want to meet the high interest and expectations of parents. Therefore they are willing to include a course, mysteriously named informatics, in the first four years of the curriculum. In most cases an informatics teacher is hired from the connected upper secondary school. This might lead to a (slightly modified) upper secondary informatics tradition in the class of 11 year old pupils.

Therefore this is an extremely challenging time for experiment. But what should such a course look like? How should it fit into current school practice so that the school accepts it? Also, inspired by Papert's visions, see [2], how do we introduce a new wind into lower secondary education? The parable of the Trojan horse came to mind: if Papert is right, computers and new approaches to integrate them into education can help to change the school.

In this paper we present an experimental course designed for a class of 11 year olds. This course has run for one school year.

\section{Initial findings}

The students turned out to have better computers at home (PC's of their parents) than at school. This lead to ambitious suggestions such as "... let us work with the C language", or "... we want to program". Others suggested playing games. All of them were sure that this course would be "about computers". And all of them immediately wanted to do BIG things because they had already seen big things. From our previous experience we know that children with such expectations can easily be disappointed by the usual educational software of often doubtful quality. All in all this was a very difficult starting position for a teacher.

From the very beginning we wanted to show that the computer, and in general information technology, is not a goal in itself, but a means to solve problems, a means to express oneself, and a means to create something. We wanted the students to understand that there are also problems which cannot be 
solved, even by a computer, and that computer use comes in many guises. We did not want to demonstrate software packages which they do not need, and to identify computer use with playing games, although we sometimes managed to make use of some commercial games in a surprising way.

The students should work with different forms of information, collect and process data with the appropriate interpretation. To scan photos, to decorate the resulting images by adding a moustache... In projects our students should develop their intellectual capabilities, algorithmic thinking and exploratory habits. They should also be made to understand the relation between sequences of events and their static descriptions in a predefined symbolic language, for example describing Logo turtle drawings by simple sentences or commands. We have presented an example of such learning environment in [3], aimed at developing learning strategies, at experimenting with new software applications and at cooperative work in a small groups.

Along the lines set out in [4] students should learn some elements of turtle geometry developing educational software in Logo. When children are convinced they are developing something of value, they are highly motivated for solving difficult problems. In the second year of lower secondary education the students are introduced to the Logo environment, turtle geometry, programming of simple procedures etc. In our view introductory Logo programming is a natural bridge between what children already know of mathematics, and some more advanced concepts, such as angles, polygons, plane and space understanding, symbolic procedural descriptions of mathematical figures and drawings (independent of their orientation.

The students experiment with several open learning environments in a project approach with no centralized teaching/learning methods throughout the full school year, an approach not very common in other subjects. The meetings with the students, for only 90 minutes a week, are organized in such a way that each activity has a kind of outcome, for example, a printout of a picture story composed this week. On the other hand many projects cover more than one week - even then children should feel they are approaching a certain goal.

Our approach is completely Windows based following the general trend to equip schools with PC Windows machines. Many Windows applications have a standard userinterface which is an advantage in an exploratory approachyou immediately know how to start, to quit, to navigate through menus, to import and export data via the Clipboard, and to use the Help facility. We are aware of the fact that this implies rather high hardware requirements. However, we believe this is a right decision because:

- to keep the old 8-bit practice would mean to petrify the tradition; 
- many new lower secondary schools cooperate with some upper secondary school and make use of their computer lab;

- many of schools are just being established these days and we are setting high standards for them.

A new implementation of Logo, Comenius Logo for Windows, is used [5]. New feature is the use of Logo in two different ways:

- as a language of turtle geometry, and as a tool for developing your own procedures;

- as an Environment for Environments.

In [6] we have described in detail that the underlying Logo is a complex tool with powerful developing and debugging features, which we call an environment. When a developed application has a complex conception (either educational or not) and nontrivial interactions, behaviour and structure, we also call this an environment. Thus, the underlying Logo, allowing development of such environments, is an environment for environments-a tool powerful enough to produce other complex applications. In [6] we have also listed several features which classify a software tool to be an environment for environments.

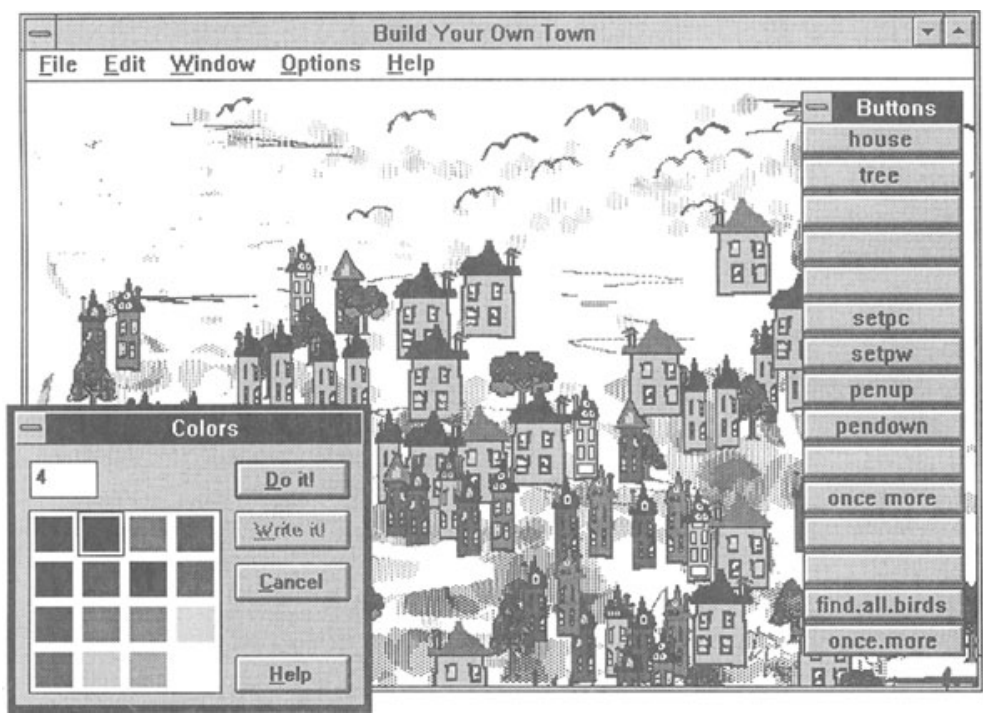

Fig. 1 Build your own town 
Within this new metaphor for Logo we and our university students have developed many open ended learning environments (microworlds) to meet the needs of children in our experimental class. Comenius Logo for Windows allows development of such powerful, open and flexible applications to be used and modified by children themselves thus actively involving them in the design work.

Environments which we are using can be divided into two groups. The first of them consists of Logo microworlds with the goal to explore and understand some concepts of Logo turtle geometry itself. A Build Your Own Town project, presented in [7], is an example of such a microworld (see Fig. $1)$.

The other group consists of projects which aim at some completely different topics. Figure 2 illustrates a project which helps to code and decode a picture by a sequence of numbers - several other secrete coding projects will be mentioned below.

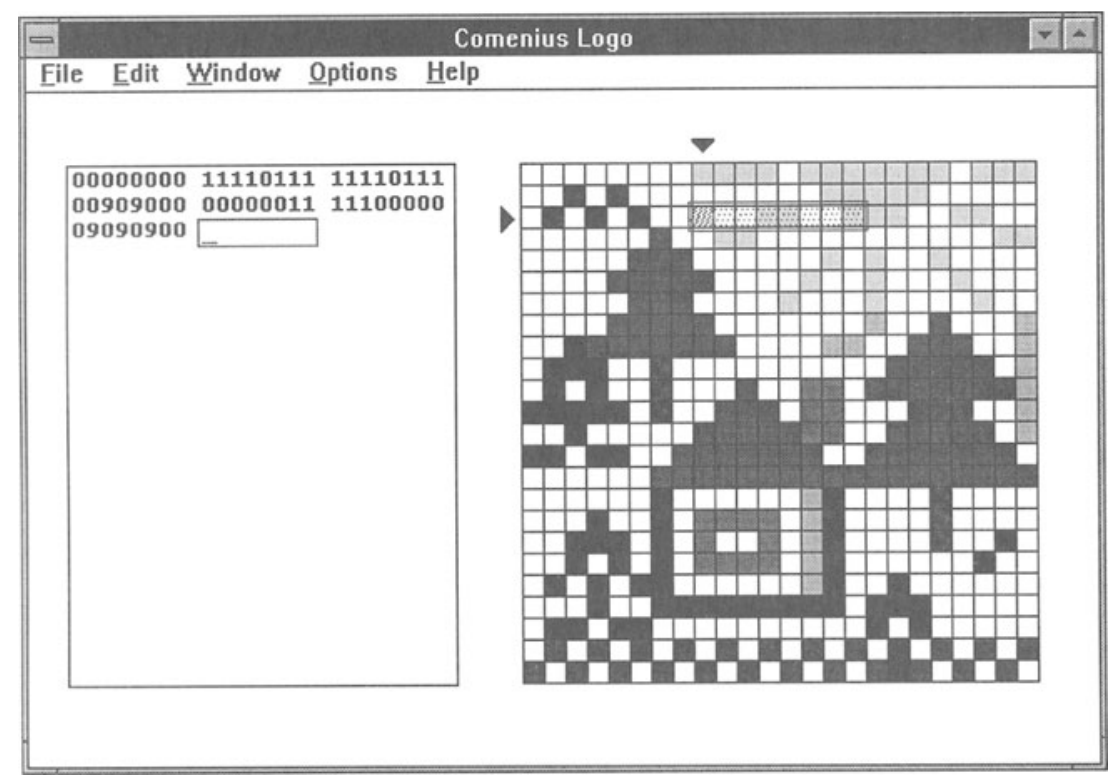

Fig. 2 Secret codes 


\section{TOPICS AND PROJECTS}

Next we will describe six general topics of our curriculum. Except for the first one, these are not in chronological order and consist of several projects of one or more weeks.

\section{Introduction to Windows}

With no special computer knowledge required, and inspired by an interesting book by Stuur [8] we started by jumping into the pool through some introductory Windows Accessories, Clock and Calculator. We experimented with starting and quitting an application, moving and resizing its window, minimizing and maximizing it, all by mouse manipulation which the children found quite easy and natural.

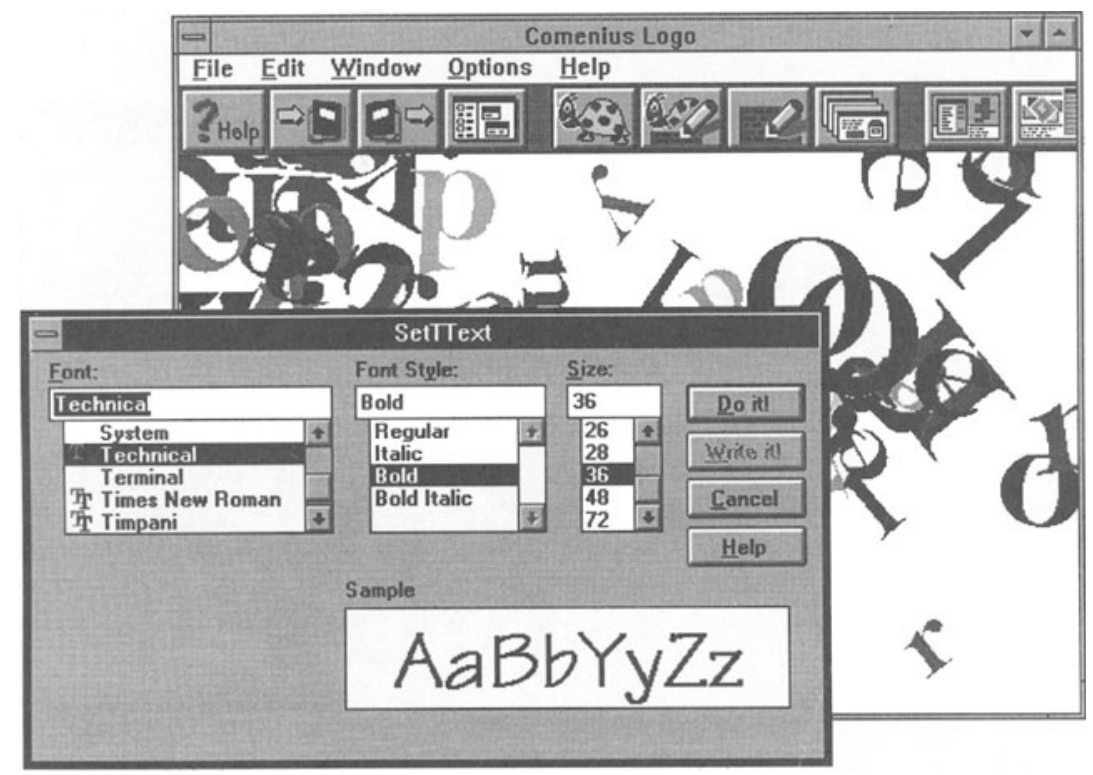

Fig. 3 Choose a font for turtle

A command was chosen from an application menu to change the mode of the clock from analogue to digital, to remove or restore the title line, etc. Also a Set Font... command was chosen; its dialogue box also contains a Sample subwindow which makes the process of changing the font much more instructive. This idea is also found in 'choosers' in Comenius Logo which students will use throughout the whole year. Choosers are special dialogue 
windows with graphical and textual information allowing choice of options like setting the turtle pen colour, its width, pattern, heading. Also the font to be used for typing into the graphics screen, see Fig. 3.

Our experience shows that the Windows environment, its structure and interface, is well designed for children users.

\section{Paintbrush: a more complex application}

Throughout the whole school year the Paintbrush graphical editor was used for many different purposes. In an experimental way children got acquainted with its tools, palette and some special commands. They learned to cut, paste and thus multiply some parts of the screen. Especially multiplying parts of a drawing presented many interesting topics.

There were several small projects, for example:

- designing an amusing imaginary business card advertising each student's own extraordinary business, like selling the hottest ice cream in town;

- designing a complete monetary system, that is, a set of one's own coins and banknotes.

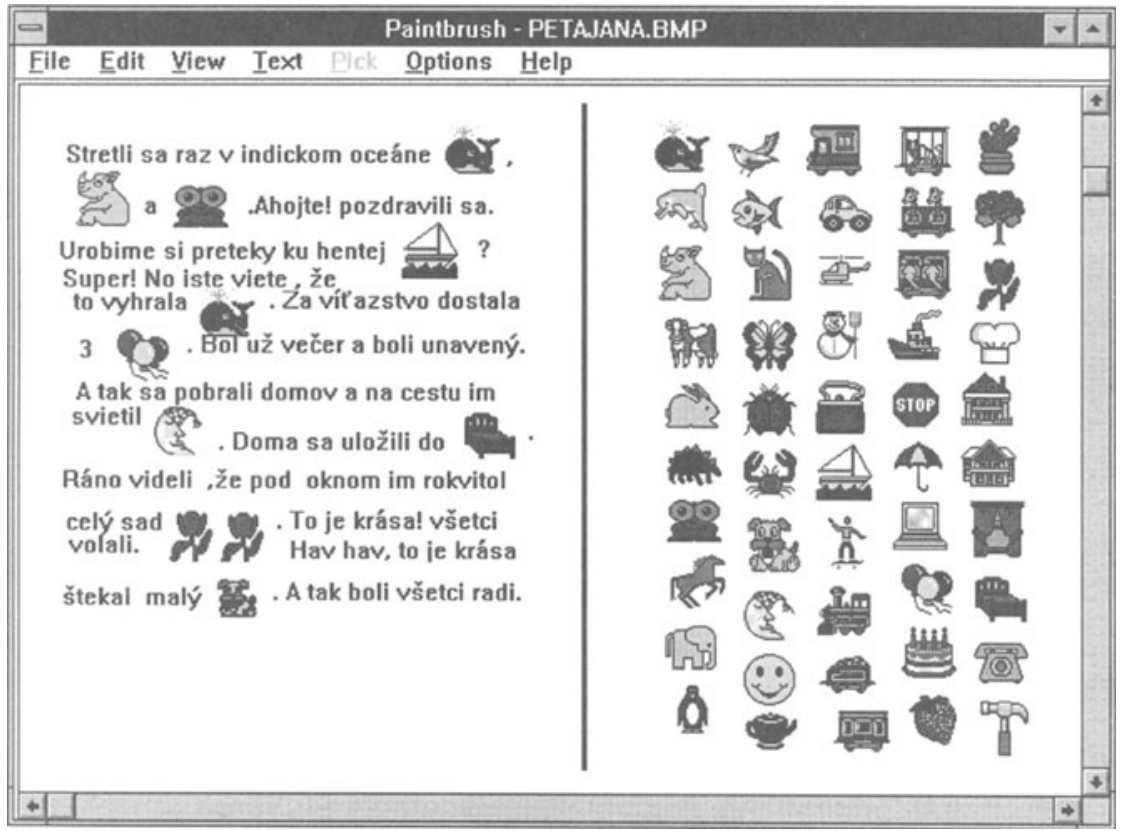

Fig. 4 Text and picture story 
In a more complex project, students were provided with a collection of small icons produced in the KidPix editor. We placed them to the right side of the screen. Students, mostly working in pairs, created a mixed text and picture story by choosing any topic, copying any icon out of this collection and combining it together with some text, (Fig. 4). Although very simple, such projects already require some planning activity and a lot of cooperation and communication among students.

\section{Secret codes and secret messages}

It is always a guaranteed success to do secret work with children of this age. Making things secret, however, needs some coding and decoding, and thus is clarified: secret code would be of no use if its addressee could not receive and understand the message.

Very different things can be coded: texts, drawings (what about sounds, movements, smells... ?). We started on paper with texts and some well known alphabets like Morse. Several new alphabets were invented and coding of a drawing in a string of digits 1 and 0 , where 1 stands for a filled square, 0 for an empty square (Fig. 5). We discussed some related problems: How to reduce the code? When decoding, how to know the number of squares in a line?

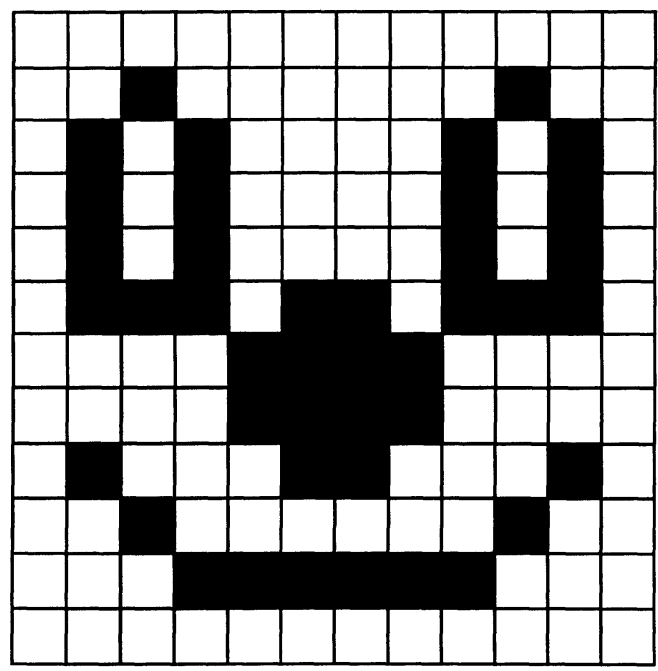

000000000000 001000000100 010100001010 010100001010 010100001010 011101101110 000011110000 000011110000 010001100010 001000000100 000111111000 000000000000

Fig. 5 Simple coding 
One way to shorten the code of a drawing is to convert each 4 subsequent binary digits into the corresponding decimal number. The same can be done with 8 digits, but this requires more calculations-is this then not the right place to use a computer for coding and decoding? Can the information on colours be included as well? Then some more complex coding applications or environments prepared in Comenius Logo are presented, see for example Fig. 2.

Coding activities illustrate fundamental issues in information processing and handling. Many interesting questions arise: Is it possible to produce a mirror image of an original drawing from its number code? Is it possible to let the drawing shrink or make it bigger? Is not a simple Logo program itself a secret code of a certain drawing describing the process of drawing? Is it difficult to code drawings into Logo programs? Is it difficult to decode or interpret such descriptions?

Other codes-Braille alphabet among them-were used to illustrate how difficult it is for blind and partially sighted children to absorb a piece of information.

\section{First contacts with Logo}

Our students meet Logo for the first time by playing with all its demo projects designed to illustrate the environment, using the Speed bar and the Buttons bar, the turtle, many turtles, and controlling Logo programs by mouse (for more information see [5].) Some of these projects are simple games for developing logical minds, others demonstrate screen animation or develop problem solving skills - all based on the Comenius Logo environment. Any teacher or any student can add his own project to the menu of the demo projects.

To prepare students for controlling a turtle they first learn to control themselves. Playing in a classroom, giving and realizing simple commands to move robots (students). Then some single line paper drawings are coded into a sequence of simple Logo like commands. Such sequences can then be decoded by other students-do both drawings match? If not, where is the mistake?

Controlling the turtle from a command line is very simple, starting with forward, right and left commands. At this point in learning we use the Build Your Own Town project described in [7]. Children build a town by navigating the turtle across the screen and using the special commands house and tree. Some other features of the project and Comenius Logo are explored mainly by themselves. They learn how effective it is to press any button in the Buttons window instead of typing in the whole command, they learn how to define their own buttons and the associated behaviour (actions). They exploit buttons even for defining they own new Logo procedures. Advance is also due to the many available Choosers (see Fig. 1). All of these tools are designed for young users and hide many technical details. 
In the Build Your Own Town project students inspect the procedures and images in Logo and modify these. They add their own houses and trees, and they try to introduce their own animations.

\section{Activities developing algorithmic thinking and logical mind}

Throughout the year a lot of small games or projects were done to develop the concept of process as a purposeful sequence of certain steps. Some of these activities were paper and pencil games, others were implemented in Comenius Logo. The main objective was to get to understand the connection between a real action and its static description. In some of these activities the children invented their own description language.

For example, the students play a simple computer game of Tic-Tac-Toe. Soon they were pleased to learn that the program could be beaten. On our suggestions their winning strategies were put on paper which required a repeatable critical sequence and its representation.

In [6] we have also presented Labyrinth, a Logo microworld aimed at developing cooperative work in problem solving. Each team of children consists of two pairs: only one of them works with a computer. Neither of the two pairs can solve the problem by itself as each pair has only partial information. Messages - sequences of simple instructions for a treasure finding robot - are being sent from one pair to another. Each partial success of one pair makes it possible for another pair to advance one step towards final solution. Our goal in this cooperative activity is to work with different representations (languages for descriptions) of the same problem.

In a Train project children are given a simple scheme of railway lines: a single track turns into double track and then at another point joins back into a single track. A train with one engine and three cars numbered 1,2 and 3 stands on the right single track and waits to be shunted. Students should find an appropriate sequence of moves so that the train ends up on the left single track reordered, for example as follows: 2 , engine, 3,1 . Hundreds of interesting assignments can be obtained if we modify the scheme of a layout by adding some more side tracks, the number of cars or engines, or any additional constraints. This microworld gives an opportunity to discuss the number of necessary moves, to discuss why some assignments could not be solved at all, or to invent a language for describing some general strategies.

In Secret Billiards, students are playing in pairs. The playing table, on a sheet of paper or on the screen, is a grid of 3 times 3 squares (see Fig. 6). Several types of small obstacles, listed on the right of the figure, can be placed inside the grid so that an imaginary ball will change its path if it hits an obstacle. The ball is always shot from outside the grid, it crosses the squares, maybe hits some obstacles and changes its heading, and finally leaves the grid somewhere. 
The first player now secretly chooses to place certain obstacles in the gridonly in his mind. Now the grid functions as a black box: the other player knows neither where the obstacles are nor of what type they are. The second player chooses any starting point for shooting a ball and asks the first player where the ball would leave the scheme. The assignment is to localise all obstacles and guess their type. How many shots will the player need?
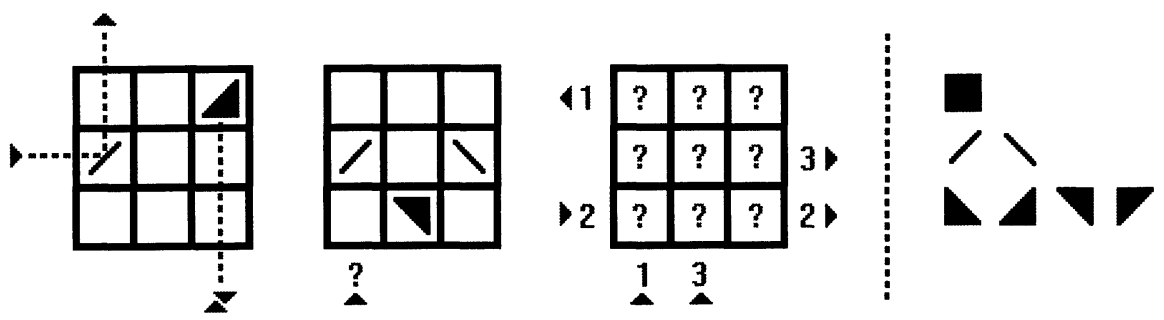

Fig. 6 Secret billiards

\section{Information technologies and information processing}

Whenever it is technically possible, we incorporate more specialized hardware. We managed to bring into our classroom a table scanner and used it for two weeks in a project for forging banknotes. Students scanned in their photo portraits and either modified, multiplied or combined them as bitmaps in the Paintbrush editor, or used them as amusing substitutes for official portraits on our banknotes. Students appreciated this project because they were working with real data-with real pictures and banknotes easily transported into their world of Windows.

The students experienced an interesting sequence of data transformations: they transformed a photo through the scanner into a data file on a floppy disk. Then they copied the file into their machine and were able to process the data.

A similar creative atmosphere was created when the students exchanged a series of e-mail messages with our university teacher students. However, due to the hardware situation, this was only a short excursion into new information technologies rather than regular project work. In a similar way we presented some other devices like a real time animation system or a computer based sound studio. 


\section{CONCLUSION}

Probably the biggest obstacles we were fighting throughout the whole school year were technical problems with computer lab maintenance, due to many divergent interests of the users of the lab. In spite of this we have been receiving serious support from the principal and from other teachers as well. The experiment is now in its second year-we hope to open our Trojan horse slightly this year, not to subvert, but to draw in some teachers of other subjects in a proper way.

\section{REFERENCES}

1. Stanchev, I., Nikolov, R. and Nikolova, I. (1994) Perspectives on Teacher Training Related to Information Technology in Central and Eastern European Countries. In Proc. of UNESCO Workshop: Teacher Education and Communication and Information Technologies, Enschede.

2. Papert, S. (1993) The Children's Machine. BasicBooks.

3. Blaho, A. and Kalas, I. (1993) Thomash the Clown's Circus: Order in Action in Picture Languages, in Informatics and Changes in Learning (eds Johnson, D. C. and Samways, B.), IFIP Transactions A-34, North-Holland, Amsterdam, pp. 79-86.

4. Harel, I. and Papert, S. (1991) Software Design as a Learning Environment, in Constructionist Learning (ed Harel, I.), MIT Media Lab, pp. 19-50.

5. Blaho, A., Kalas, I. and Tomcsanyi, P. (1993) Comenius Logo: Environment for Teachers and Environment for Learners, in Proc. of 4th EuroLogo Conference, Athens, Supplement, pp. 1-11.

6. Blaho, A., Kalas, I. and Matusova, M. (1994) Environment for Environments: A New Metaphor for Logo, in Exploring a New Partnership: Children, Teachers and Technology (eds Wright J. and Benzie, D.), IFIP Transactions A-58, North-Holland, Amsterdam, pp. 153-166. 
7. Kalas, I. and Blaho, A. (1994) Not All Birds Are Turtles. Developing Teaching/Learning Environments in Initial Teacher Training at Comenius University, in Proc. of UNESCO Workshop: Teacher Education and Communication and Information Technologies, Enschede.

8. Stuur, A. (1993) Windows Voor Kinderen. A.W. Bruna Informatica, Utrecht. 